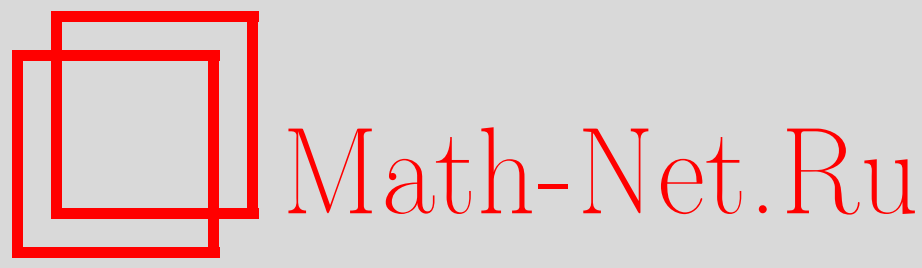

И. Я. Арефьева, И. В. Волович, С. В. Козырев, Метод стохастического предела и интерференция в квантовых многочастичных системах, ТМФ, 2015, том 183, номер 3, 388-408

DOI: https://doi.org/10.4213/tmf8828

Использование Общероссийского математического портала Math-Net.Ru подразумевает, что вы прочитали и согласны с пользовательским соглашением http://www . mathnet.ru/rus/agreement

Параметры загрузки:

IP: 34.229 .108 .108

26 апреля 2023 г., 17:16:12

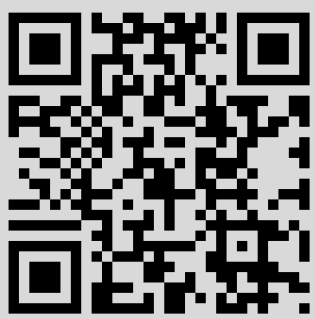




\title{
МЕТОД СТОХАСТИЧЕСКОГО ПРЕДЕЛА И ИНТЕРФЕРЕНЦИЯ В КВАНТОВЫХ МНОГОЧАСТИЧНЫХ СИСТЕМАХ
}

\begin{abstract}
Для многочастичных квантовых систем с дипольным взаимодействием с квантовым полем рассмотрена проблема транспорта энергии электронного возбуждения при помощи механизма экситонного переноса, основанного на квантовой интерференции. Показано, что при специальном выборе параметров взаимодействия в модели квантового графа можно достичь усиления переноса экситона на сток, пропорционального числу частиц в системе, а также подавить перенос на альтернативные стоки. Для описания динамики используется метод квантового стохастического предела. Отмечены возможные применения указанного механизма в процессах квантового фотосинтеза.
\end{abstract}

Ключевые слова: стохастический предел квантовой теории, динамика многочастичных квантовых систем, процессы квантового переноса.

DOI: $10.4213 / \operatorname{tmf} 8828$

\section{1. ВВЕДЕНИЕ}

Проблеме переноса энергии электронного возбуждения в сложных квантовых системах посвящено большое число работ. В частности, широко используется метод стохастического предела [1], теория Фёрстера-Декслера и ее различные обобщения (см. монографию [2]). Стохастический предел для взаимодействия двухуровневого атома с полем рассматривался в работе [3]. Многочастичные квантовые системы в стохастическом пределе исследовались в статьях [4], [5]. В последнее время привлекли большое внимание замечательные открытия эффективной передачи энергии при квантовом фотосинтезе в некоторых биологических системах (см. обзоры в [6], [7]).

В настоящей работе для многочастичных квантовых систем с дипольным взаимодействием с квантовым полем рассматривается модель транспорта энергии электронного возбуждения при помощи механизма экситонного переноса, основанного на квантовой интерференции.

Мы обсуждаем проблему переноса экситона на сток в одноэкситонном приближении, т. е. распад состояния от одноэкситонного к невозбужденному через стоки.

* Математический институт им. В. А. Стеклова РАН, Москва, Россия. E-mail: arefeva@mi.ras.ru, volovich@mi.ras.ru, kozyrev@mi.ras.ru 
В качестве метода исследования динамики квантовых систем мы применяем приближение стохастического предела квантовой теории [1], т. е. приближение квантовой динамики некоторым квантовым случайным процессом. Многочастичные квантовые системы в стохастическом пределе исследовались, в частности, в работе [4]. В стохастическом пределе мы изучаем динамику системы, взаимодействующей с окружением (резервуаром), который описывается квантовым бозонным полем. Также мы описываем стоки (поглощения экситонов) при помощи дополнительных резервуаров.

Подход настоящей работы отличается от обычно применяемого в подобных обсуждениях также в следующем. Во-первых, передача возбуждения между разными квантовым состояниями является стандартным поведением в квантовых взаимодействующих системах. Поэтому мы должны показать для рассматриваемой модели не только возможность передачи возбуждения на сток, но и высокую эффективность такой передачи. Во-вторых, поглощение экситона может происходить в разных состояниях системы, т. е. система может иметь много стоков. Мы должны объяснить, почему это поглощение происходит на одном стоке (притом с повышенной скоростью), а процессы поглощения на остальных стоках подавлены. Причиной такого эффекта может быть квантовая интерференция.

Мы рассматриваем модель, гамильтониан которой отвечает некоторому квантовому графу, т. е. графу, вершины которого нумеруют векторы ортонормированного базиса в (конечномерном) гильбертовом пространстве, а ребрам сопоставлены некоторые комплексные числа (связанные со скоростями перехода между такими состояниями). Мы рассматриваем граф с вершинами $|0\rangle,|1\rangle, \ldots,|N\rangle,|N+1\rangle, \ldots,|N+M\rangle$. Здесь $|0\rangle$ есть состояние без экситона, остальные вершины образуют базис в одноэкситонном секторе, при этом вершины $|N+1\rangle, \ldots,|N+M\rangle$ отвечают стоку экситона. Ребра графа отвечают некоторым комплексным числам $g_{i j}$, по которым строится гамильтониан модели (вершины $i$ и $j$ соединены ребром, если $g_{i j} \neq 0$ ).

Мы рассматриваем задачу Коши для квантовой динамики экситона с начальным условием в виде экситона $|1\rangle$. Мы показываем, что можно подобрать квантовый граф (величи́ны $g_{i j}$ ) таким образом, чтобы сток шел через состояние $|N+1\rangle$ и не шел через состояния $|j\rangle$ с номерами $j=N+2, \ldots, N+M$. Подобное поведение можно получить за счет квантовой интерференции в многочастичных системах. Отметим, что такая математическая модель может найти применение при теоретическом объяснении недавно открытых экспериментально наблюдаемых эффектов квантовой когерентности в фотосинтетических комплексах [8]-[10]. Для модели, развиваемой в настоящей работе, существенным является наличие когерентностей для состояний второго слоя квантового графа. Когерентности такого сорта наблюдались в упомянутых экспериментах.

Полученные результаты можно сравнить с результатами работы [11] и использованным в указанной работе видом линдбладовских генераторов динамики (разделением генераторов на диссипацию, дефазинг и сток). В рамках подхода работы [11] существенную роль играет часть генератора, называемая дефазингом. В методе стохастического предела такая часть генератора отсутствует. Механизм экситонного транспорта, описанный в настоящей работе, существенно отличается от подхода работы [11]. 
Структура настоящей работы имеет следующий вид.

В разделе 2 мы приводим вид генератора динамики матрицы плотности, получающегося в методе стохастического предела для квантовых многочастичных систем с дипольным взаимодействием. В разделе 3 мы обсуждаем вид гамильтониана изучаемой модели экситонного переноса и соответствующего квантового графа. Гамильтониан данной многочастичной системы рассматривается как вырожденный он имеет только четыре уровня энергии, два из них сильно вырожденны. В разделе 4 мы приводим вид генератора динамики для матрицы плотности системы из раздела 3 в стохастическом пределе квантовой теории. Подобные генераторы для систем с вырождением могут описывать интерференцию. В разделе 5 приведены простейшие примеры, а в разделе 6 мы изучаем квантовую динамику, отвечающую переносу экситонов, в общем случае и показываем, что в рассматриваемой модели за счет квантовой интерференции усиливается транспорт на один из стоков, а альтернативные стоки полностью подавляются. В заключении мы обсуждаем результаты, полученные в настоящей работе.

\section{2. ГЕНЕРАТОРЫ, ОТВЕЧАЮЩИЕ КВАНТОВЫМ ГРАФАМ}

В этом разделе мы рассматриваем генераторы динамики для матрицы плотности, получающиеся в методе стохастического предела для системы многих частиц с дипольным взаимодействием с квантовым полем. Изложенные в настоящем разделе подходы можно найти в работе [4].

Гамильтониан системы имеет конечное число собственных векторов,

$$
H_{\mathrm{S}}=\sum_{i=1}^{N} \varepsilon_{i}|i\rangle\langle i|,
$$

где среди энергий $\varepsilon_{i}$ могут быть совпадающие. Также может быть вырожден спектр оператора $\left[H_{\mathrm{S}}, \cdot\right]$, т. е. одной боровской частоте (разности между двумя уровнями энергии) могут отвечать переходы между разными уровнями энергии.

Пусть система взаимодействует с резервуаром - бозонным квантовым полем со свободным гамильтонианом

$$
H_{\mathrm{R}}=\int \omega(k) a^{*}(k) a(k) d k
$$

в гауссовском состоянии

$$
\left\langle a^{*}(k) a\left(k^{\prime}\right)\right\rangle=N(k) \delta\left(k-k^{\prime}\right)
$$

с нулевым средним. Здесь $a^{*}(k), a\left(k^{\prime}\right)$ - стандартные бозонные операторы рождения и уничтожения в $\mathbb{R}^{3}$, а функцию $\omega(k)$ можно выбрать, например, в виде $\omega(k)=k^{2} / 2$. $\mathrm{B}$ частности, для гиббсовского (температурного) состояния

$$
N(k)=\frac{1}{e^{\beta \omega(k)}-1},
$$

где $\beta>0$ - обратная температура. Для фоковского состояния $N(k)=0$. 
Гамильтониан взаимодействия $H_{\text {I }}$ системы с полем имеет вид

$$
H_{\mathrm{I}}=\sum_{i, j=1}^{N}\left(g_{i j}^{*} A(g)|i\rangle\left\langle j\left|+g_{i j} A^{*}(g)\right| j\right\rangle\langle i|\right), \quad A^{*}(g)=\int g(k) a^{*}(k) d k,
$$

где $g_{i j}$ суть комплексные числа и $g(k)$ - основная функция. Мы считаем $g_{i j}=g_{j i}$ для всех $i, j$. Таким образом, мы предположили, что формфакторы гамильтониана взаимодействия между различными парами состояний отличаются умножением на числа.

Гамильтониан, являющийся суммой трех вышеперечисленных гамильтонианов, системы, резервуара и взаимодействия,

$$
H=H_{\mathrm{S}}+H_{\mathrm{R}}+\lambda H_{\mathrm{I}},
$$

мы будем называть гамильтонианом квантового графа (здесь $\lambda>0$ есть константа связи). Вершинами графа являются состояния системы (собственные векторы $|i\rangle$, $i=1, \ldots, N$, гамильтониана системы), ребра отвечают числам $g_{i j}$ и описывают взаимодействия между состояниями системы через посредство резервуара. Мы будем говорить, что в квантовом графе существует ребро между вершинами $i$ и $j$, если соответствующий коэффициент $g_{i j}$ отличен от нуля.

Стохастический предел состоит в перерастяжке времени $t \rightarrow t / \lambda^{2}$ и рассмотрении квантовой динамики в пределе $\lambda \rightarrow 0$. При этом квантовая динамика будет приближаться некоторым квантовым случайным процессом [1]. Случай стохастического предела для квантовых многочастичных систем и соответствующая динамика матриц плотности изучались в работе [4].

Динамика матрицы плотности в стохастическом пределе задается уравнением

$$
\frac{d}{d t} \rho(t)=\theta(\rho(t))
$$

где генератор разлагается в сумму по боровским частотам $\omega$,

$$
\theta=\sum_{\omega} \theta_{\omega}
$$

а каждая $\theta_{\omega}$ является суммой диссипативной и осцилляторной части:

$$
\begin{aligned}
& \theta_{\omega}(\rho)= \theta_{\omega}^{\text {diss }}(\rho)+\theta_{\omega}^{\text {osc }}(\rho)= \\
&=\sum_{a, b} \sum_{c, d} g_{a b}^{*} g_{c d}\left(2 \operatorname{Re}(g \mid g)_{\omega}^{-}\left(\langle c|\rho| a\rangle|d\rangle\langle b|-\frac{1}{2} \delta_{b d}\{\rho,|a\rangle\langle c|\}\right)+\right. \\
& \quad+2 \operatorname{Re}(g \mid g)_{\omega}^{+}\left(\langle b|\rho| d\rangle|a\rangle\langle c|-\frac{1}{2} \delta_{a c}\{\rho,|d\rangle\langle b|\}\right)- \\
&\left.\quad-i \operatorname{Im}(g \mid g)_{\omega}^{-} \delta_{b d}[\rho,|a\rangle\langle c|]+i \operatorname{Im}(g \mid g)_{\omega}^{+} \delta_{a c}[\rho,|d\rangle\langle b|]\right)
\end{aligned}
$$


Здесь

$$
\begin{aligned}
(g \mid g)_{\omega}^{+} & =-i \int|g(k)|^{2} \frac{1}{\omega(k)-\omega-i 0} N(k) d k, \\
(g \mid g)_{\omega}^{-} & =-i \int|g(k)|^{2} \frac{1}{\omega(k)-\omega-i 0}(N(k)+1) d k, \\
\operatorname{Re}(g \mid g)_{\omega}^{+} & =\pi \int|g(k)|^{2} \delta(\omega(k)-\omega) N(k) d k, \\
\operatorname{Re}(g \mid g)_{\omega}^{-} & =\pi \int|g(k)|^{2} \delta(\omega(k)-\omega)(N(k)+1) d k, \\
\operatorname{Im}(g \mid g)_{\omega}^{+} & =-\int|g(k)|^{2} \mathrm{P} . \mathrm{V} \cdot\left\{\frac{1}{\omega(k)-\omega}\right\} N(k) d k, \\
\operatorname{Im}(g \mid g)_{\omega}^{-} & =-\int|g(k)|^{2} \mathrm{P} \cdot \mathrm{V} \cdot\left\{\frac{1}{\omega(k)-\omega}\right\}(N(k)+1) d k,
\end{aligned}
$$

где $\omega=\varepsilon(a)-\varepsilon(b)=\varepsilon(c)-\varepsilon(d)$ есть боровская частота, $\varepsilon(a)$ - энергия состояния $a$. Суммирование в (4) идет по парам состояний $a$ и $b, c$ и $d$, отвечающим боровской частоте $\omega$. Специфика квантового графа (набор ребер) в выражении для генератора находит свое отражение в коэффициентах $g_{i j}$. Диссипативная часть генератора (содержащая константы $\left.\operatorname{Re}(g \mid g)_{\omega}^{ \pm}\right)$может быть отлична от нуля только для положительных боровских частот.

Для системы общего положения (когда каждой боровской частоте отвечают ровно два уровня энергии с разностью, равной этой боровской частоте) число вкладов в генератор будет расти линейно с ростом числа $N$ уровней системы. Для систем с вырождением, например, когда все переходы отвечают всего одной боровской частоте, возможен более быстрый рост генератора как функции от $N$ [4]. Мы увидим, что этот эффект естественно связывать с эффективностью экситонного транспорта при квантовом фотосинтезе.

\section{3. ГАМИЛЬТОНИАН МОДЕЛИ}

Для описания квантового фотосинтеза рассмотрим систему в $(N+M+1)$-мерном гильбертовом пространстве с ортонормированным базисом $|0\rangle,|1\rangle, \ldots,|N+M\rangle$. Такая система будет описывать молекулярный комплекс в одноэкситонном приближении, вектор $|0\rangle$ отвечает отсутствию возбуждения (экситона), остальные векторы образуют базис в одноэкситонном секторе. Мы будем рассматривать для такой системы взаимодействие между состояниями, происходящее через посредство бозонного квантового поля (резервуара).

Гамильтониан системы (без учета взаимодействия с резервуаром) имеет вид

$$
H_{\mathrm{S}}=\varepsilon_{1}|1\rangle\left\langle 1\left|+\varepsilon_{2} \sum_{j=2}^{N}\right| j\right\rangle\left\langle j\left|+\varepsilon_{3} \sum_{j=N+1}^{N+M}\right| j\right\rangle\langle j| .
$$

При этом все боровские частоты $\omega=\varepsilon_{i}-\varepsilon_{j}$, отвечающие энергиям $\varepsilon_{0}=0, \varepsilon_{1}, \varepsilon_{2}, \varepsilon_{3}$, между собой различны и $\varepsilon_{1}>\varepsilon_{2}>\varepsilon_{3}>0$.

Состояния, отвечающие фиксированной энергии для такого гамильтониана, мы будем называть слоями квантового графа (см. рис. 1). Таким образом, мы имеем четыре слоя: первый слой (с энергией $\varepsilon_{1}$ ) содержит состояние $|1\rangle$, второй слой 


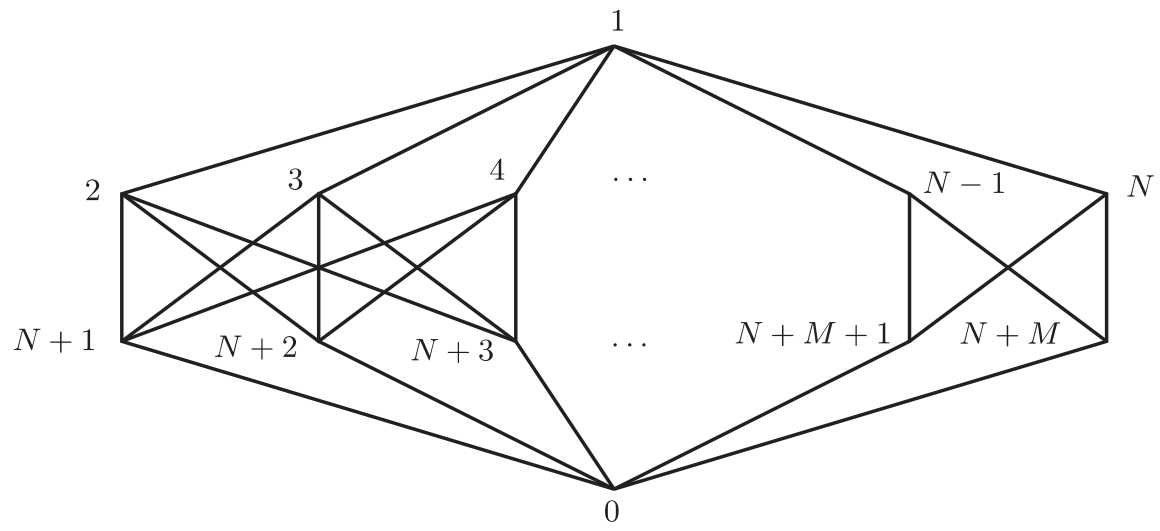

Рис. 1. Квантовый граф состояний системы.

(с энергией $\varepsilon_{2}$ ) содержит состояния $|2\rangle, \ldots,|N\rangle$, третий слой (с энергией $\varepsilon_{3}$ ) содержит состояния $|N+1\rangle, \ldots,|N+M\rangle$, четвертый слой (с энергией $\left.\varepsilon_{0}=0\right)$ содержит состояние $|0\rangle$. Мы будем считать, что число стоков не превосходит числа состояний в промежуточном слое квантового графа, т. е. $M<N$.

Мы считаем, что вершины $|N+1\rangle, \ldots,|N+M\rangle$ третьего слоя квантового графа являются стоками, т. е. система из них переходит в состояние $|0\rangle$ (без экситона).

Мы также считаем, что взаимодействия между вершинами квантового графа происходят посредством бозонного поля. Система взаимодействует с $M+1$ резервуарами (бозонными квантовыми полями) через дополнительные гамильтонианы взаимодействия. Резервуары описываются гамильтонианами

$$
\begin{gathered}
H_{\mathrm{R}}=\int \omega_{\mathrm{R}}(k) a^{*}(k) a(k) d k, \\
H_{\mathrm{r}}=\sum_{j=N+1}^{N+M} H_{\mathrm{r}}^{(j)}, \quad H_{\mathrm{r}}^{(j)}=\int \omega_{\mathrm{r}}(k) b_{j}^{*}(k) b_{j}(k) d k, \quad j=N+1, \ldots, N+M .
\end{gathered}
$$

Первый резервуар описывает колебательные степени свободы окружения и порождает переходы между состояниями одноэкситонного сектора. Состояние поля $a(k)$ описывается некоторым гауссовским состоянием (например, температурным) с квадратичным коррелятором (2). Остальные резервуары описывают стоки. Физический смысл выделения стока в отдельный резервуар состоит в том, что это естественно экситон в стоке уходит за счет взаимодействия с полем, описывающим окружение. Мы выбираем много независимых резервуаров, потому что нам удобно считать стоки независимыми. Это означает отсутствие квантовых корреляций при стоке на каждом из таких состояний. Отвечающие стокам резервуары суть бозонные поля в фоковских (вакуумных) состояниях, гамильтониан взаимодействия для стоков имеет вид

$$
H_{\mathrm{i}}=\sum_{j=N+1}^{N+M}\left(B_{j}+B_{j}^{*}\right)(|0\rangle\langle j|+| j\rangle\langle 0|), \quad B_{j}^{*}=\int h(k) b_{j}^{*}(k) d k .
$$


Гамильтониан взаимодействия $H_{\mathrm{I}}$ для резервуара $H_{\mathrm{R}}$ имеет вид $(3)$, где суммирование пробегает по состояниям одноэкситонного сектора от $|1\rangle$ до $|N+M\rangle$. Для изучаемой модели мы рассматриваем квантовый граф для $H_{\mathrm{I}}$ с ненулевыми ребрами $g_{i j}$ в случае переходов между состояниями $|1\rangle$ и $|2\rangle, \ldots,|N\rangle$, а также между состояниями $|2\rangle, \ldots,|N\rangle$ и $|N+1\rangle, \ldots,|N+M\rangle$ (т. е. взаимодействия имеют место для всех вершин из соседних слоев квантового графа).

Определим квантовый граф, задающий изучаемую систему. Важную роль будет играть условие интерференции

$$
\sum_{a=2}^{N} g_{a b}=0, \quad b=N+2, \ldots, N+M .
$$

Для этого мы выбираем коэффициенты графа как

$$
\begin{array}{ll}
g_{1 j}=1, & j=2, \ldots, N, \\
g_{a b}=e^{2 \pi i a(b-N-1) /(N-1)}, & a=2, \ldots, N, \quad b=N+1, \ldots, N+M .
\end{array}
$$

В частности, $g_{a, N+1}=1$ для $a=2, \ldots, N$. Остальные коэффициенты квантового графа положим равными нулю.

Суммарный гамильтониан системы и окружения имеет вид

$$
H=H_{\mathrm{S}}+H_{\mathrm{R}}+H_{\mathrm{r}}+\lambda H_{\mathrm{I}}+\lambda H_{\mathrm{i}},
$$

где $\lambda>0$ есть константа связи.

\section{4. ГЕНЕРАТОР ДИНАМИКИ МОДЕЛИ}

В соответствии с подходом стохастического предела к многочастичным системам с дипольным взаимодействием (см. раздел 2) генератор динамики матрицы плотности для изучающейся в настоящей работе системы имеет вид

$$
\theta=\theta_{1}+\theta_{2}+\theta_{3},
$$

где первые два вклада в генератор отвечают взаимодействию системы с колебательными степенями свободы (с резервуаром (2)) для двух боровских частот $\omega_{1}=\varepsilon_{1}-\varepsilon_{2}$ и $\omega_{2}=\varepsilon_{2}-\varepsilon_{3}$. Первая часть генератора $\theta_{1}$ отвечает переходам между первым и вторым уровнями квантового графа, т. е. переходам между начальным состоянием $|1\rangle$ и вершинами $|2\rangle, \ldots,|N\rangle$, и боровской частоте $\omega_{1}$. Вторая часть генератора $\theta_{2}$ отвечает переходам между вторым и третьим уровнями квантового графа, т. е. переходам между вершинами $|2\rangle, \ldots,|N\rangle$ и состояниями стока $|N+1\rangle, \ldots,|N+M\rangle$, и боровской частоте $\omega_{2}$. Часть $\theta_{3}$ генератора, соответствующая стокам с полями $b_{j}$, является суммой членов, совпадающих с генератором для двухуровневого атома (спин-бозонная модель [3]), и отвечает боровской частоте $\varepsilon_{3}$.

Генераторы $\theta_{1}$ и $\theta_{2}$ имеют вид

$$
\begin{aligned}
\theta_{1}(\rho) & =\sum_{\substack{a=1, \ldots, N, b=2, \ldots, N}} \sum_{\substack{c=1, \ldots, N, d=2, \ldots, N}} g_{a b}^{*} g_{c d}\left(2 \gamma_{\mathrm{re}}^{-}\left(\langle c|\rho| a\rangle|d\rangle\langle b|-\frac{1}{2} \delta_{b d}\{\rho,|a\rangle\langle c|\}\right)+\right. \\
& \left.+2 \gamma_{\mathrm{re}}^{+}\left(\langle b|\rho| d\rangle|a\rangle\langle c|-\frac{1}{2} \delta_{a c}\{\rho,|d\rangle\langle b|\}\right)-i \gamma_{\mathrm{im}}^{-} \delta_{b d}[\rho,|a\rangle\langle c|]+i \gamma_{\mathrm{im}}^{+} \delta_{a c}[\rho,|d\rangle\langle b|]\right)=
\end{aligned}
$$




$$
\begin{aligned}
= & \sum_{b, d=2}^{N}\left(2 \gamma_{\mathrm{re}}^{-}\left(\langle 1|\rho| 1\rangle|d\rangle\langle b|-\frac{1}{2} \delta_{b d}\{\rho,|1\rangle\langle 1|\}\right)+2 \gamma_{\mathrm{re}}^{+}\left(\langle b|\rho| d\rangle|1\rangle\langle 1|-\frac{1}{2}\{\rho,|d\rangle\langle b|\}\right)-\right. \\
& \left.-i \gamma_{\mathrm{im}}^{-} \delta_{b d}[\rho,|1\rangle\langle 1|]+i \gamma_{\mathrm{im}}^{+}[\rho,|d\rangle\langle b|]\right) .
\end{aligned}
$$

Здесь

$$
\begin{gathered}
\gamma_{\mathrm{re}}^{ \pm}=\operatorname{Re}(g \mid g)_{\omega_{1}}^{ \pm}, \quad \gamma_{\mathrm{im}}^{ \pm}=\operatorname{Im}(g \mid g)_{\omega_{1}}^{ \pm}, \\
\omega_{1}=\varepsilon(a)-\varepsilon(b)=\varepsilon(c)-\varepsilon(d)=\varepsilon_{1}-\varepsilon_{2} .
\end{gathered}
$$

Аналогично,

$$
\begin{aligned}
& \theta_{2}(\rho)=\sum_{\substack{a=2, \ldots, N, b=N+1, \ldots, N+M}} \sum_{\substack{c=2, \ldots, N, d=N+1, \ldots, N+M}} g_{a b}^{*} g_{c d}\left(2 \eta_{\mathrm{re}}^{-}\left(\langle c|\rho| a\rangle|d\rangle\langle b|-\frac{1}{2} \delta_{b d}\{\rho,|a\rangle\langle c|\}\right)+\right. \\
& \left.+2 \eta_{\mathrm{re}}^{+}\left(\langle b|\rho| d\rangle|a\rangle\langle c|-\frac{1}{2} \delta_{a c}\{\rho,|d\rangle\langle b|\}\right)-i \eta_{\mathrm{im}}^{-} \delta_{b d}[\rho,|a\rangle\langle c|]+i \eta_{\mathrm{im}}^{+} \delta_{a c}[\rho,|d\rangle\langle b|]\right)= \\
& =\sum_{\substack{a=2, \ldots, N, b=N+1, \ldots, N+M}} \sum_{\substack{c=2, \ldots, N, d=N+1, \ldots, N+M}} e^{-2 \pi i a(b-N-1) /(N-1)} e^{2 \pi i c(d-N-1) /(N-1)} \times \\
& \times\left(2 \eta_{\mathrm{re}}^{-}\left(\langle c|\rho| a\rangle|d\rangle\langle b|-\frac{1}{2} \delta_{b d}\{\rho,|a\rangle\langle c|\}\right)+2 \eta_{\mathrm{re}}^{+}\left(\langle b|\rho| d\rangle|a\rangle\langle c|-\frac{1}{2} \delta_{a c}\{\rho,|d\rangle\langle b|\}\right)-\right. \\
& \left.-i \eta_{\mathrm{im}}^{-} \delta_{b d}[\rho,|a\rangle\langle c|]+i \eta_{\mathrm{im}}^{+} \delta_{a c}[\rho,|d\rangle\langle b|]\right) .
\end{aligned}
$$

Здесь боровская частота имеет вид $\omega_{2}=\varepsilon_{2}-\varepsilon_{3}$, соответствующие коэффициенты в генераторе обозначены как

$$
\eta_{\mathrm{re}}^{ \pm}=\operatorname{Re}(g \mid g)_{\omega_{2}}^{ \pm}, \quad \eta_{\mathrm{im}}^{ \pm}=\operatorname{Im}(g \mid g)_{\omega_{2}}^{ \pm}
$$

Генератор $\theta_{3}$ примет вид суммы по стокам:

$$
\begin{aligned}
\theta_{3}(\rho)=\sum_{j=N+1}^{M} & \left(i \operatorname{Im}(h \mid h)_{\omega}^{-}[\rho,|j\rangle\langle j|]+i \operatorname{Im}(h \mid h)_{-\omega}^{-}[\rho,|0\rangle\langle 0|]+\right. \\
& \left.+2 \operatorname{Re}(h \mid h)_{\omega}^{-}\left(\langle j|\rho| j\rangle|0\rangle\langle 0|-\frac{1}{2}\{\rho,|j\rangle\langle j|\}\right)\right) .
\end{aligned}
$$

Здесь боровская частота $\omega$ принимает значение $\varepsilon_{3}$, и мы учли, что для вакуумного состояния $(h \mid h)_{\omega}^{+}=0$.

\section{5. ДИНАМИКА МОДЕЛИ В ПРОСТЕЙШИХ СЛУЧАЯХ}

В настоящем разделе мы разберем динамику введенной выше модели в двух простейших случаях, а именно в случае, когда каждый слой квантового графа содержит по одному состоянию (случай без вырождения), и в случае, когда мы имеем двукратное вырождение во втором слое квантового графа (см. рис. 2). Все отличные от нуля коэффициенты $g_{a b}$ квантового графа, заданные в (6), в этих случаях будут равны единице. 

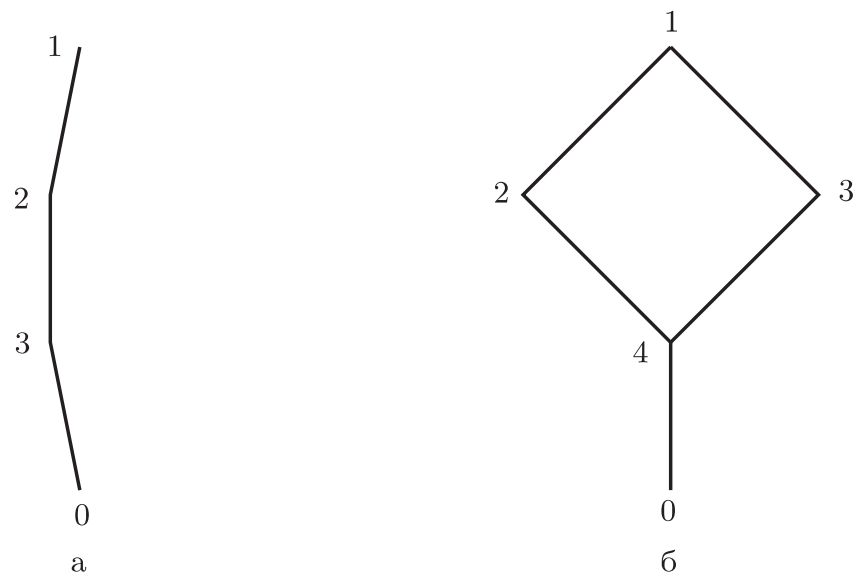

Рис. 2. Графы состояний в простейших случаях

5.1. Случай без вырождения. В первом случае (без вырождения) система будет системой общего положения. Динамика таких систем обсуждалась в работах [1], [4]. Вклады в генератор принимают вид

$$
\begin{aligned}
\theta_{1}(\rho)= & 2 \gamma_{\mathrm{re}}^{-}\left(\rho_{11}|2\rangle\langle 2|-\frac{1}{2}\{\rho,|1\rangle\langle 1|\}\right)+ \\
& +2 \gamma_{\mathrm{re}}^{+}\left(\rho_{22}|1\rangle\langle 1|-\frac{1}{2}\{\rho,|2\rangle\langle 2|\}\right)-i \gamma_{\mathrm{im}}^{-}[\rho,|1\rangle\langle 1|]+i \gamma_{\mathrm{im}}^{+}[\rho,|2\rangle\langle 2|], \\
\theta_{2}(\rho)= & 2 \eta_{\mathrm{re}}^{-}\left(\rho_{22}|3\rangle\langle 3|-\frac{1}{2}\{\rho,|2\rangle\langle 2|\}\right)+ \\
& +2 \eta_{\mathrm{re}}^{+}\left(\rho_{33}|2\rangle\langle 2|-\frac{1}{2}\{\rho,|3\rangle\langle 3|\}\right)-i \eta_{\mathrm{im}}^{-}[\rho,|2\rangle\langle 2|]+i \eta_{\mathrm{im}}^{+}[\rho,|3\rangle\langle 3|], \\
\theta_{3}(\rho)= & -i \operatorname{Im}(h \mid h)_{\omega}^{-}[\rho,|3\rangle\langle 3|]-i \operatorname{Im}(h \mid h)_{-\omega}^{-}[\rho,|0\rangle\langle 0|]+ \\
& +2 \operatorname{Re}(h \mid h)_{\omega}^{-}\left(\rho_{33}|0\rangle\langle 0|-\frac{1}{2}\{\rho,|3\rangle\langle 3|\}\right) .
\end{aligned}
$$

Для таких генераторов внедиагональная часть матрицы плотности будет экспоненциально убывать, потому что внедиагональные элементы будут собственными векторами генератора с отрицательной вещественной частью. При подстановке матрицы плотности в генератор $\theta=\theta_{1}+\theta_{2}+\theta_{3}$ мы получим ненулевой вклад в действие генератора, пропорциональный $|1\rangle\langle 2|$, который имеет вид

$$
\theta_{1}(|1\rangle\langle 2|)=\left(-\gamma_{\mathrm{re}}^{-}-\gamma_{\mathrm{re}}^{+}+i \gamma_{\mathrm{im}}^{-}+i \gamma_{\mathrm{im}}^{+}-\eta_{\mathrm{re}}^{-}-i \eta_{\mathrm{im}}^{-}\right)|1\rangle\langle 2| .
$$

Аналогичное поведение будет иметь место для других внедиагональных элементов матрицы плотности. Таким образом, для внедиагональных элементов матрицы 
плотности мы получим

$$
\begin{aligned}
& \frac{d}{d t} \rho_{12}(t)=\left(-\gamma_{\mathrm{re}}^{-}-\gamma_{\mathrm{re}}^{+}+i \gamma_{\mathrm{im}}^{-}+i \gamma_{\mathrm{im}}^{+}-\eta_{\mathrm{re}}^{-}-i \eta_{\mathrm{im}}^{-}\right) \rho_{12}(t), \\
& \frac{d}{d t} \rho_{13}(t)=\left(-\gamma_{\mathrm{re}}^{-}+i \gamma_{\mathrm{im}}^{-}-\eta_{\mathrm{re}}^{+}+i \eta_{\mathrm{im}}^{+}-i \operatorname{Im}(h \mid h)_{\omega}^{-}-\operatorname{Re}(h \mid h)_{\omega}^{-}\right) \rho_{13}(t), \\
& \frac{d}{d t} \rho_{23}(t)=\left(-\gamma_{\mathrm{re}}^{+}-i \gamma_{\mathrm{im}}^{+}-\eta_{\mathrm{re}}^{-}-\eta_{\mathrm{re}}^{+}+i \eta_{\mathrm{im}}^{-}+i \eta_{\mathrm{im}}^{+}-i \operatorname{Im}(h \mid h)_{\omega}^{-}-\operatorname{Re}(h \mid h)_{\omega}^{-}\right) \rho_{23}(t) .
\end{aligned}
$$

Отсюда следует, что внедиагональные элементы матрицы плотности исчезают в пределе больших времен. Такое поведение отвечает явлению декогеренции.

Диагональные элементы матрицы плотности будут эволюционировать отдельно от внедиагональных. Для диагональных элементов матрицы плотности мы получим систему уравнений

$$
\begin{aligned}
& \frac{d}{d t} \rho_{11}(t)=-2 \gamma_{\mathrm{re}}^{-} \rho_{11}(t)+2 \gamma_{\mathrm{re}}^{+} \rho_{22}(t), \\
& \frac{d}{d t} \rho_{22}(t)=2 \gamma_{\mathrm{re}}^{-} \rho_{11}(t)-2 \gamma_{\mathrm{re}}^{+} \rho_{22}(t)-2 \eta_{\mathrm{re}}^{-} \rho_{22}(t)+2 \eta_{\mathrm{re}}^{+} \rho_{33}(t), \\
& \frac{d}{d t} \rho_{33}(t)=2 \eta_{\mathrm{re}}^{-} \rho_{22}(t)-2 \eta_{\mathrm{re}}^{+} \rho_{33}(t)-2 \operatorname{Re}(h \mid h)_{\epsilon_{3}}^{-} \rho_{33}(t), \\
& \frac{d}{d t} \rho_{00}(t)=2 \operatorname{Re}(h \mid h)_{\epsilon_{3}}^{-} \rho_{33}(t) .
\end{aligned}
$$

Все коэффициенты $\gamma_{\mathrm{re}}^{ \pm}, \eta_{\mathrm{re}}^{ \pm}, \operatorname{Re}(h \mid h)_{\epsilon_{3}}^{-}$в этой системе неотрицательны.

Рассмотрим случай, когда функции $g(k)$ и $h(k)$ зависят только от $k^{2}$. Тогда, используя формулы (5) и (7), получим

$$
\begin{gathered}
\operatorname{Re}(g \mid g)_{\omega}^{+}=\pi \int|g(k)|^{2} \delta(\omega(k)-\omega) N(k) d k=\frac{C}{e^{\beta \omega}-1}, \\
\operatorname{Re}(g \mid g)_{\omega}^{-}=\pi \int|g(k)|^{2} \delta(\omega(k)-\omega)(N(k)+1) d k=\frac{C}{1-e^{-\beta \omega}}, \\
\gamma_{\mathrm{re}}^{-}=\frac{C}{1-e^{-\beta \omega_{1}}}, \quad \eta_{\mathrm{re}}^{-}=\frac{C}{1-e^{-\beta \omega_{2}}}, \\
\gamma_{\mathrm{re}}^{+}=e^{-\beta \omega_{1}} \gamma_{\mathrm{re}}^{-}, \quad \eta_{\mathrm{re}}^{+}=e^{-\beta \omega_{2}} \eta_{\mathrm{re}}^{-} \\
\operatorname{Re}(h \mid h)_{\epsilon_{3}}^{-}=\frac{C_{1}}{1-e^{-\beta \epsilon_{3}}},
\end{gathered}
$$

где $C, C_{1}$ - неотрицательные константы, не зависящие от $\beta$. Уравнения (15) следуют из соотношений Эйнштейна. Здесь для $\operatorname{Re}(h \mid h)^{-}$в (16) взято температурное распределение. Для фоковского состояния $\operatorname{Re}(h \mid h)^{-}=C_{1}$.

Отметим, что если положить $C_{1}=0$, то гиббсовское состояние

$$
\rho_{i i}=\text { const } \cdot e^{-\beta \varepsilon_{i}}, \quad i=1,2,3,
$$

является стационарным решением уравнений (11).

Таким образом, система уравнений (8)-(11) будет описывать термализацию состояний $|1\rangle,|2\rangle,|3\rangle$ в результате взаимодействия с температурным резервуаром (отвечающим колебательным степеням свободы), а взаимодействие состояний $|3\rangle$ и $|0\rangle$ 


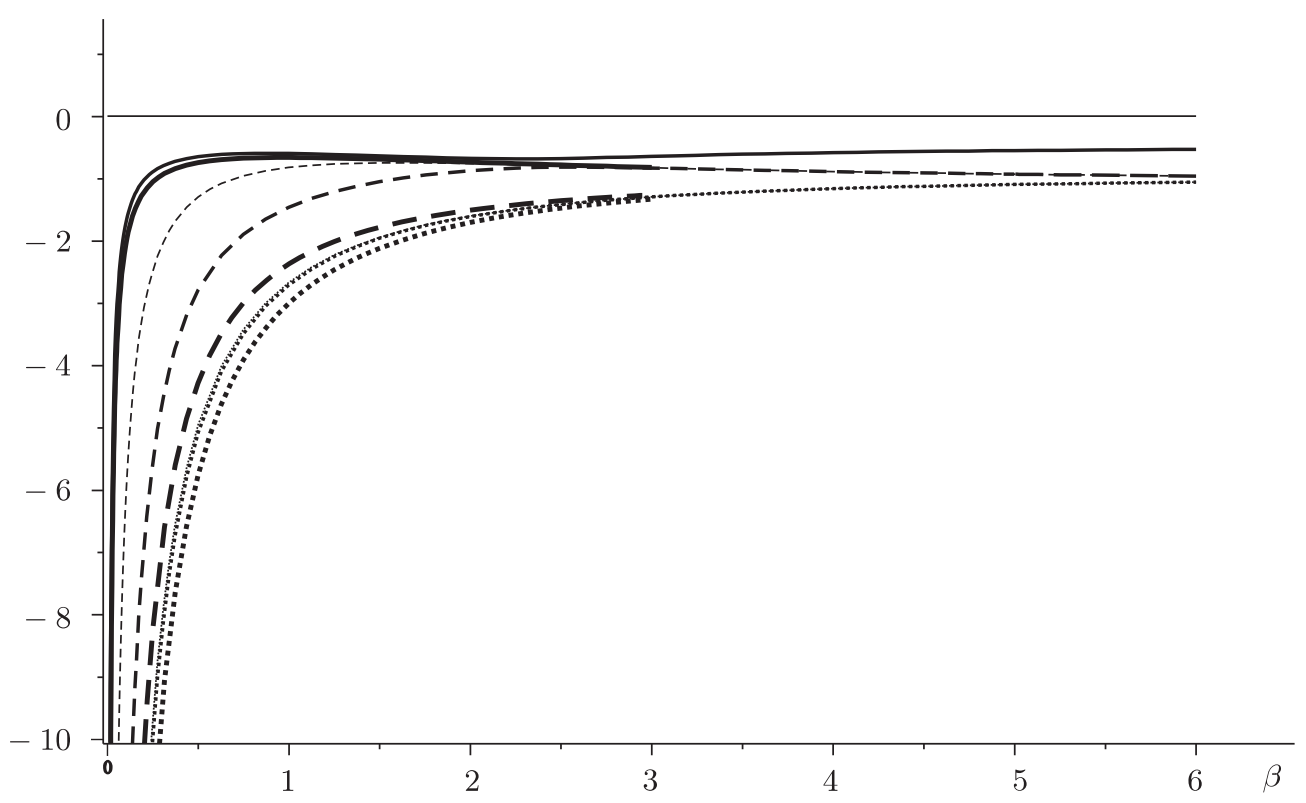

Рис. 3. Зависимость собственных значений $\lambda_{i}$ (на рисунке $i=1-$ сплошные линии, $i=2$ - штриховые линии, $i=3$ - пунктирные линии) от обратной температуры $\beta$ при некоторых фиксированных энергиях $\varepsilon_{i}, i=1,2,3$, при $C=1$ и различных значениях $C_{1}$. Различные значения $C_{1}$ соответствуют различной толщине кривых: $C_{1}=0$ - тонкие линии, $C_{1}=0.5$ - линии средней толщины, $C_{1}=1$ - толстые линии.

(описывающееся отдельным резервуаром в фоковском состоянии) будет отвечать стоку экситона. В результате динамика будет состоять в уходе возбуждений в сток. Можно обратить внимание на то, что скорости декогеренции и термализации управляются одними и теми же параметрами.

Отметим, что имеется интересная зависимость решений уравнений (11) от температуры $T=1 / \beta$. Для обсуждения этой зависимости заметим, что первые три уравнения системы (11) могут быть записаны как

$$
\frac{d}{d t} \Psi(t)=M \Psi(t), \quad \Psi(t)=\left(\begin{array}{l}
\rho_{11}(t) \\
\rho_{22}(t) \\
\rho_{33}(t)
\end{array}\right), \quad M=\left(\begin{array}{ccc}
-a & b & 0 \\
a & -b-c & d \\
0 & c & -d-f
\end{array}\right)
$$

где

$$
a=2 \gamma_{\mathrm{re}}^{-}, \quad b=2 \gamma_{\mathrm{re}}^{+}, \quad c=2 \eta_{\mathrm{re}}^{-}, \quad d=2 \eta_{\mathrm{re}}^{+}, f=2 \operatorname{Re}(h \mid h)_{\epsilon_{3}}^{-} .
$$

Зависимость собственных значений $\lambda_{i}, i=1,2,3$, матрицы $M$ от обратной температуры $\beta$ при некоторых фиксированных энергиях $\varepsilon_{i}, i=1,2,3$, при $C=1$ и различных значениях $C_{1}$ приведена на рис. $3-5$. На рис. 5 видна немонотонная зависимость $\lambda_{1}$ от $\beta$ при $C_{1} \neq 0$. При уменьшении температуры (увеличении значения $\beta$ ) значение $\lambda_{1}$ сначала быстро увеличивается, достигает максимума и затем уменьшается. В этом смысле можно говорить о наличии динамического фазового перехода. 

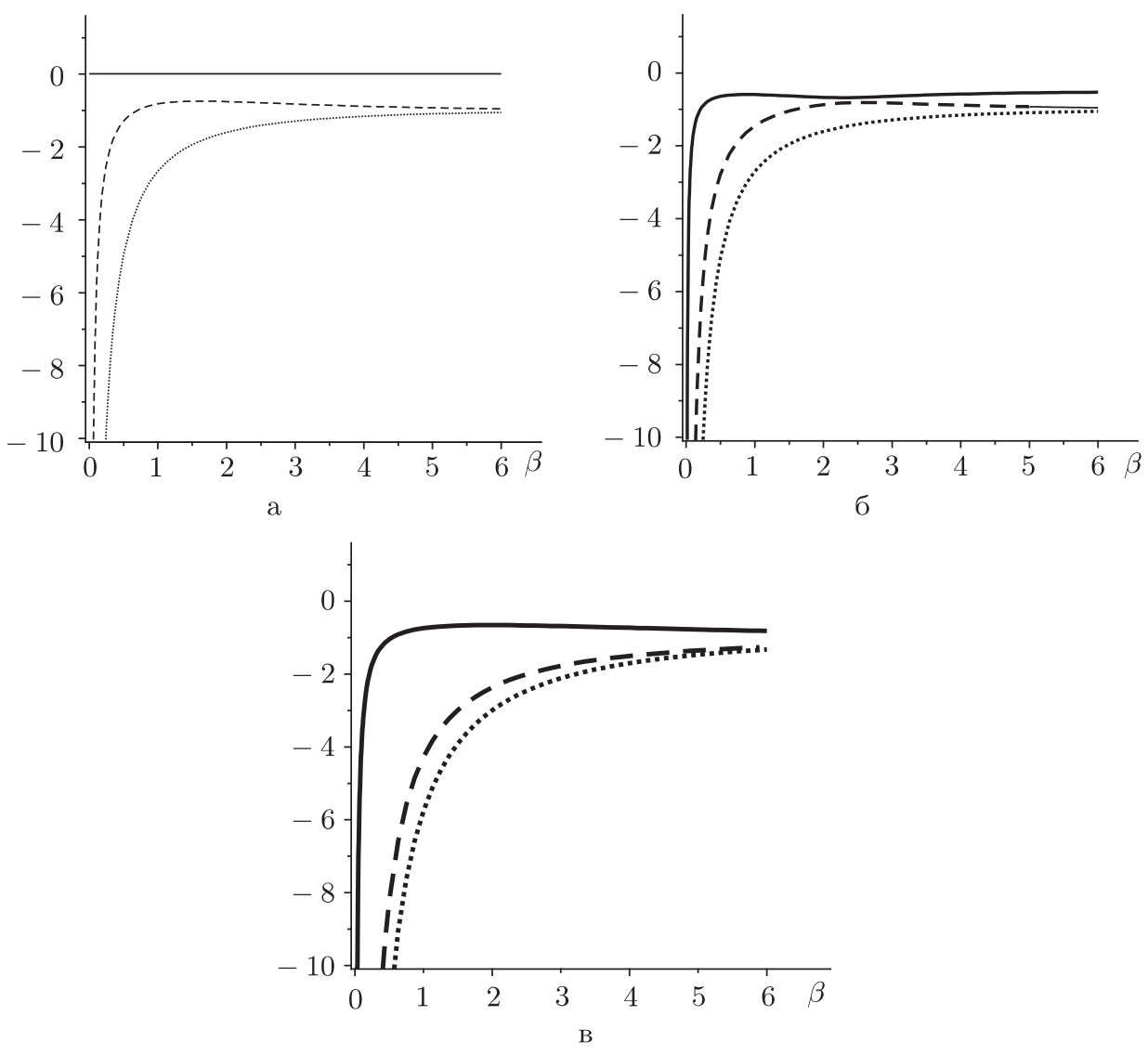

Рис. 4. То же, что и на рис. 3, отдельно для значений $C_{1}=0$ (a), $C_{1}=0.5$ (б), $C_{1}=1$ (в). Виден максимум значения $\lambda_{2}$ на первом и третьем рисунках.

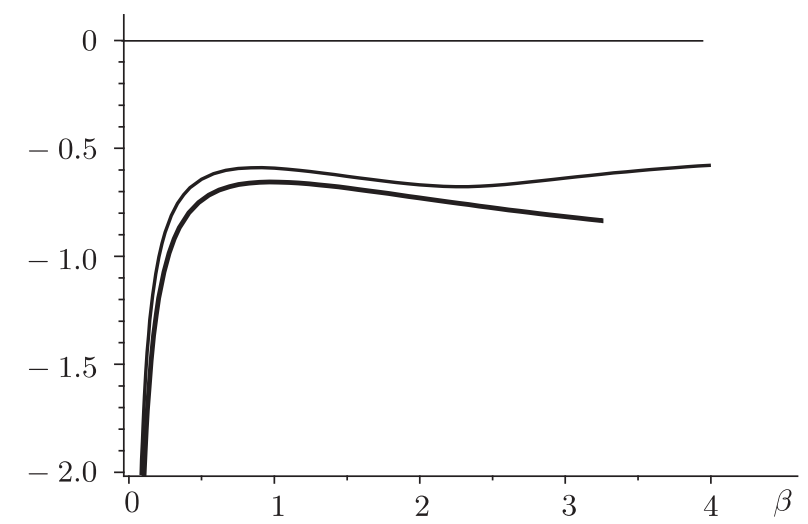

Рис. 5. Зависимость величины $\lambda_{1}$ от обратной температуры. Различные значения $C_{1}$ соответствуют различной толщине кривых: $C_{1}=0$ - тонкие линии, $C_{1}=0.5$ - линии средней толщины, $C_{1}=1$ - толстые линии. 
На рис. 3 и 4 видна немонотонная зависимость $\lambda_{2}$ от $\beta$ при не слишком больших $C_{1}$, а также и при $C_{1}=0$ (см. рис. $\left.4 \mathrm{a}\right)$.

Зависимость $\lambda_{2}$ от обратной температуры при $C_{1}=0$ видна из явной формулы для $\lambda_{2}$. Действительно, в этом случае $\lambda_{1}=0$ и два оставшихся корня характеристического уравнения имеют вид

$$
\lambda_{2,3}=-\frac{1}{2}(a+b+c+d) \pm \sqrt{K}
$$

где $K=(a+b-c-d)^{2}+4 b c$. Оба корня отрицательные и $\lambda_{2}>\lambda_{3}$.

5.2. Функция Ляпунова для системы без вырождения. Покажем, что для системы общего положения матрица плотности (удовлетворяющая мастер-уравнению для диагональной части матрицы плотности) сходится к гиббсовскому состоянию. Внедиагональные компоненты матрицы плотности будут убывать экспоненциально. Для исследования динамики диагональной части матрицы плотности построим функционал Ляпунова (такой функционал будет похож на энтропию).

Положим $n(j)=\rho_{j j}(t)$. Мастер-уравнение для диагональной части матрицы плотности перепишем в виде (вытекающем из соотношений Эйнштейна)

$$
\frac{d n(j)}{d t}=\sum_{r, s}\left(\delta_{j s}-\delta_{j r}\right) J_{r s}\left(e^{\beta \varepsilon_{r}} n(r)-e^{\beta \varepsilon_{s}} n(s)\right),
$$

где $J_{r s} \geqslant 0$ при $\varepsilon_{r}>\varepsilon_{s}$ и $J_{r s}=0$ в противном случае.

Рассмотрим функционал

$$
S[n]=\sum_{j}\left(n(j) \ln \left(e^{\beta \varepsilon_{j}} n(j)\right)-n(j)\right)=\operatorname{tr}(\rho \ln \rho-\rho+\rho \beta H) .
$$

Пользуясь мастер-уравнением, вычислим производную по времени:

$$
\begin{aligned}
\frac{d}{d t} S[n] & =\sum_{j}\left(\frac{d n(j)}{d t} \ln e^{\beta \varepsilon_{j}} n(j)\right)= \\
& =\sum_{r, s} J_{r s}\left(e^{\beta \varepsilon_{r}} n(r)-e^{\beta \varepsilon_{s}} n(s)\right) \sum_{j}\left(\delta_{j s}-\delta_{j r}\right) \ln \left(e^{\beta \varepsilon_{j}} n(j)\right)= \\
& =\sum_{r, s} J_{r s}\left(e^{\beta \varepsilon_{r}} n(r)-e^{\beta \varepsilon_{s}} n(s)\right) \ln \frac{e^{\beta \varepsilon_{s}} n(s)}{e^{\beta \varepsilon_{r}} n(r)} .
\end{aligned}
$$

Такой функционал всегда неположителен и обращается в нуль на гиббсовском состоянии (когда $n(j)$ пропорциональны $e^{-\beta \varepsilon_{j}}$ ). Других нулей у функционала нет, если достаточно большое число коэффициентов $J_{r s}$ отличны от нуля (т. е. если квантовый граф системы является связным, точнее, содержит подграф, все ребра которого отвечают переходам с ненулевыми $\left.J_{r s}\right)$. Чтобы иметь функционал Ляпунова, нужна еще ограниченность такого функционала; для конечных систем это можно считать выполненным. 
5.3. Случай с вырождением. Простейший случай системы с вырождением в стохастическом пределе был разобран в работе [5]. Для второго примера (с вырождением) на рис. $2 б$ мы получим следующую систему мастер-уравнений для матрицы плотности:

$$
\begin{aligned}
\theta_{1}(\rho)= & \sum_{b, d=2,3}\left(2 \gamma_{\mathrm{re}}^{-}\left(\rho_{11}|d\rangle\langle b|-\frac{1}{2} \delta_{b d}\{\rho,|1\rangle\langle 1|\}\right)+2 \gamma_{\mathrm{re}}^{+}\left(\rho_{b d}|1\rangle\langle 1|-\frac{1}{2}\{\rho,|d\rangle\langle b|\}\right)-\right. \\
& \left.-i \gamma_{\mathrm{im}}^{-} \delta_{b d}[\rho,|1\rangle\langle 1|]+i \gamma_{\mathrm{im}}^{+}[\rho,|d\rangle\langle b|]\right), \\
\theta_{2}(\rho)= & \sum_{a, c=2,3}\left(2 \eta_{\mathrm{re}}^{-}\left(\rho_{c a}|4\rangle\langle 4|-\frac{1}{2}\{\rho,|a\rangle\langle c|\}\right)+2 \eta_{\mathrm{re}}^{+}\left(\rho_{44}|a\rangle\langle c|-\frac{1}{2} \delta_{a c}\{\rho,|4\rangle\langle 4|\}\right)-\right. \\
& \left.-i \eta_{\mathrm{im}}^{-}[\rho,|a\rangle\langle c|]+i \eta_{\mathrm{im}}^{+} \delta_{a c}[\rho,|4\rangle\langle 4|]\right), \\
\theta_{3}(\rho)= & -i \operatorname{Im}(h \mid h)_{\omega}^{-}[\rho,|4\rangle\langle 4|]-i \operatorname{Im}(h \mid h)_{-\omega}^{-}[\rho,|0\rangle\langle 0|]+ \\
& +2 \operatorname{Re}(h \mid h)_{\omega}^{-}\left(\rho_{44}|0\rangle\langle 0|-\frac{1}{2}\{\rho,|4\rangle\langle 4|\}\right) .
\end{aligned}
$$

Для внедиагональных матричных элементов матрицы плотности (кроме $\rho_{23}, \rho_{32}$ ) мы получим аналогичные (8) уравнения, описывающие экспоненциальное убывание таких матричных элементов.

Оставшиеся уравнения для матрицы плотности примут вид

$$
\begin{aligned}
& \frac{d}{d t} \rho_{11}(t)=-4 \gamma_{\mathrm{re}}^{-} \rho_{11}(t)+2 \gamma_{\mathrm{re}}^{+} \sum_{b, d=2,3} \rho_{b d}(t), \\
& \frac{d}{d t} \rho_{22}(t)=2 \gamma_{\mathrm{re}}^{-} \rho_{11}(t)-2 \gamma_{\mathrm{re}}^{+} \rho_{22}(t)-\gamma_{\mathrm{re}}^{+} \rho_{23}(t)-\gamma_{\mathrm{re}}^{+} \rho_{32}(t)+i \gamma_{\mathrm{im}}^{+} \rho_{23}(t)-i \gamma_{\mathrm{im}}^{+} \rho_{32}(t)+ \\
& \quad+2 \eta_{\mathrm{re}}^{+} \rho_{44}(t)-2 \eta_{\mathrm{re}}^{-} \rho_{22}(t)-\eta_{\mathrm{re}}^{-} \rho_{23}(t)-\eta_{\mathrm{re}}^{-} \rho_{32}(t)-i \eta_{\mathrm{im}}^{-} \rho_{23}(t)+i \eta_{\mathrm{im}}^{-} \rho_{32}(t), \\
& \frac{d}{d t} \rho_{33}(t)=2 \gamma_{\mathrm{re}}^{-} \rho_{11}(t)-2 \gamma_{\mathrm{re}}^{+} \rho_{33}(t)-\gamma_{\mathrm{re}}^{+} \rho_{23}(t)-\gamma_{\mathrm{re}}^{+} \rho_{32}(t)+i \gamma_{\mathrm{im}}^{+} \rho_{32}(t)-i \gamma_{\mathrm{im}}^{+} \rho_{23}(t)+ \\
& \quad+2 \eta_{\mathrm{re}}^{+} \rho_{44}(t)-2 \eta_{\mathrm{re}}^{-} \rho_{33}(t)-\eta_{\mathrm{re}}^{-} \rho_{23}(t)-\eta_{\mathrm{re}}^{-} \rho_{32}(t)-i \eta_{\mathrm{im}}^{-} \rho_{32}(t)+i \eta_{\mathrm{im}}^{-} \rho_{23}(t), \\
& \frac{d}{d t} \rho_{23}(t)=2 \gamma_{\mathrm{re}}^{-} \rho_{11}(t)-2 \gamma_{\mathrm{re}}^{+} \rho_{23}(t)-\gamma_{\mathrm{re}}^{+} \rho_{22}(t)-\gamma_{\mathrm{re}}^{+} \rho_{33}(t)+i \gamma_{\mathrm{im}}^{+} \rho_{22}(t)-i \gamma_{\mathrm{im}}^{+} \rho_{33}(t)+ \\
& \quad+2 \eta_{\mathrm{re}}^{+} \rho_{44}(t)-2 \eta_{\mathrm{re}}^{-} \rho_{23}(t)-\eta_{\mathrm{re}}^{-} \rho_{22}(t)-\eta_{\mathrm{re}}^{-} \rho_{33}(t)-i \eta_{\mathrm{im}}^{-} \rho_{22}(t)+i \eta_{\mathrm{im}}^{-} \rho_{33}(t), \\
& \frac{d}{d t} \rho_{32}(t)=2 \gamma_{\mathrm{re}}^{-} \rho_{11}(t)-2 \gamma_{\mathrm{re}}^{+} \rho_{32}(t)-\gamma_{\mathrm{re}}^{+} \rho_{22}(t)-\gamma_{\mathrm{re}}^{+} \rho_{33}(t)-i \gamma_{\mathrm{im}}^{+} \rho_{22}(t)+i \gamma_{\mathrm{im}}^{+} \rho_{33}(t)+ \\
& \quad+2 \eta_{\mathrm{re}}^{+} \rho_{44}(t)-2 \eta_{\mathrm{re}}^{-} \rho_{32}(t)-\eta_{\mathrm{re}}^{-} \rho_{22}(t)-\eta_{\mathrm{re}}^{-} \rho_{33}(t)+i \eta_{\mathrm{im}}^{-} \rho_{22}(t)-i \eta_{\mathrm{im}}^{-} \rho_{33}(t), \\
& \frac{d}{d t} \rho_{44}(t)=2 \eta_{\mathrm{re}}^{-} \sum_{a, c=2,3} \rho_{\mathrm{ca}}(t)-4 \eta_{\mathrm{re}}^{+} \rho_{44}(t)-2 \operatorname{Re}(h \mid h)_{\omega}^{-} \rho_{44}(t), \\
& \frac{d}{d t} \rho_{00}(t)=2 \operatorname{Re}(h \mid h)_{\omega}^{-} \rho_{44}(t) .
\end{aligned}
$$


Динамику такой системы можно исследовать, принимая во внимание неравенства $\gamma_{\mathrm{re}}^{ \pm}>0, \eta_{\mathrm{re}}^{ \pm}>0, \operatorname{Re}(h \mid h)_{\omega}^{-}>0$ и соотношения (14). Перепишем эту систему уравнений в виде

$$
\frac{d}{d t} X=A X
$$

где

$$
\begin{array}{rlrl}
\rho_{11}(t) & \equiv X_{1}, & \rho_{22}(t) \equiv X_{2}, & \rho_{33}(t) \equiv X_{3}, \\
\rho_{23}(t) \equiv X_{4}, & \rho_{32}(t) \equiv X_{5}, & \rho_{44}(t) \equiv X_{6},
\end{array}
$$

матрица $A$ имеет вид

$$
\left(\begin{array}{cccccc}
-4 a & 2 b-2 c & 2 b & 2 b & 2 b & 0 \\
2 a & -2 b-2 c & 0 & -b-c+i g-i k & -c-b-i g+i k & 2 d \\
2 a & 0 & -2 b-2 c & -c-b-i g+i k & -b-c+i g-i k & 2 d \\
2 a & -b-c+i g-i k & -c-b-i g+i k & -2 b-2 c & 0 & 2 d \\
2 a & -c-b-i g+i k & -b-c+i g-i k & 0 & -2 b-2 c & 2 d \\
0 & 2 c & 2 c & 2 c & 2 c & -4 d-2 f
\end{array}\right)
$$

и

$$
\begin{array}{ccc}
\gamma_{\mathrm{re}}^{-}=a, & \gamma_{\mathrm{re}}^{+}=b, & \gamma_{\mathrm{im}}^{+}=g, \\
\eta_{\mathrm{re}}^{+}=c, & \eta_{\mathrm{re}}^{+}=d, \quad & \eta_{\mathrm{im}}^{-}=k, \\
\operatorname{Re}(h \mid h)_{\omega}^{-}=f . &
\end{array}
$$

Характеристический многочлен матрицы $A$ может быть представлен в виде

$$
\operatorname{det}(A-\lambda E)=\lambda m_{1}(\lambda) m_{2}(\lambda)
$$

где

$$
\begin{aligned}
m_{1}(\lambda)= & \lambda^{2}+4 b \lambda+4 b^{2}+4 c \lambda+8 c b+4 c^{2}-8 g k+4 g^{2}+4 k^{2}, \\
m_{2}(\lambda)= & 4 \lambda^{2} b+8 f b \lambda+16 d b \lambda+8 f c \lambda+16 a d \lambda+8 a f \lambda+20 a c \lambda+40 a f c+2 f \lambda^{2}+ \\
& +4 d \lambda^{2}+16 a d c+4 c \lambda^{2}+4 a \lambda^{2}+\lambda^{3} .
\end{aligned}
$$

Имеется собственное значение $\lambda_{1}=0$; корни уравнения $m_{1}(\lambda)=0$ суть

$$
\lambda_{2}=-2 b-2 c-2 i g+2 i k, \quad \lambda_{3}=-2 b-2 c+2 i g-2 i k,
$$

а корни уравнения $m_{2}(\lambda)=0$ можно найти по формуле для корней кубического уравнения.

На рис. 6 и 7 представлена зависимость вещественной и мнимой частей корней $\lambda_{i}, i=2,3,4,5,6$ от $\beta$. На рисунках видна немонотонная зависимость от $\beta$, как и в предыдущем случае системы без вырождения. Кроме этого, мы можем заметить, что $\operatorname{Re} \lambda_{2}=\operatorname{Re} \lambda_{3}, \operatorname{Im} \lambda_{2}=-\operatorname{Im} \lambda_{3}$ при значениях $k=1, g=2$, а также $\operatorname{Im} \lambda_{2}=\operatorname{Im} \lambda_{3}=\operatorname{Im} \lambda_{3}$ при $k=2, g=2$.

В следующем разделе динамика исследуется в более общем случае (для генератора, отвечающего квантовому графу на рис. 1).

Наличие корня $\lambda_{1}=0$ означает, что имеется неравновесное стационарное состояние (темное состояние, dark state в квантовой оптике). При наличии вырождения 


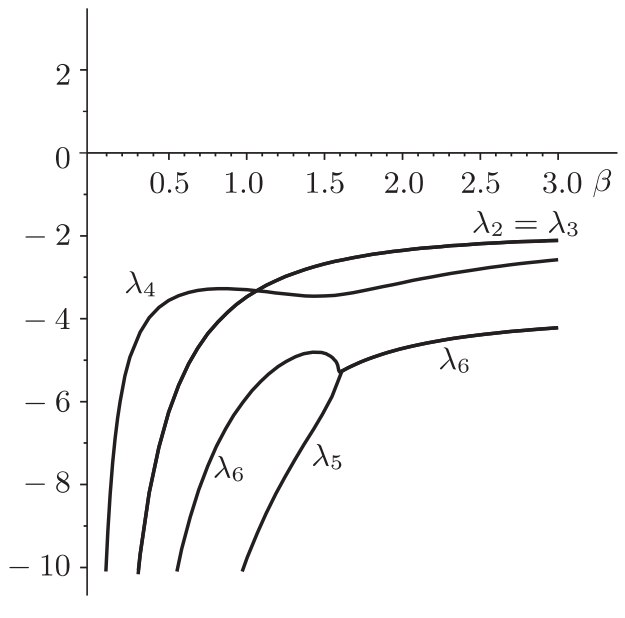

a

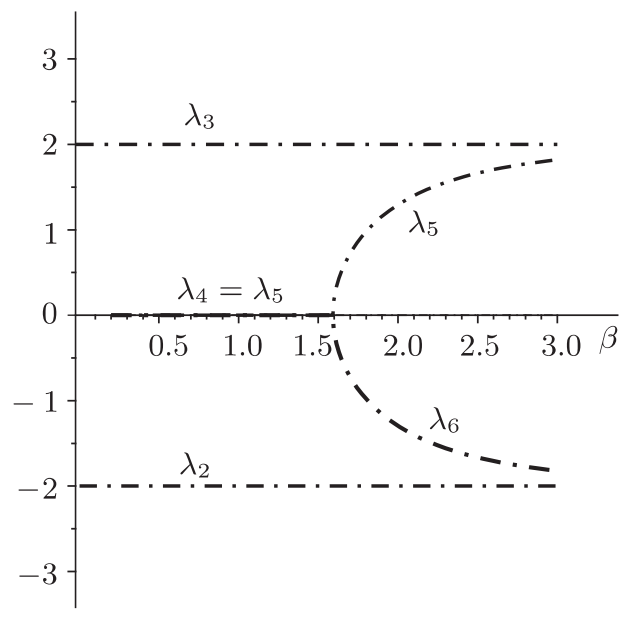

б

Рис. 6. Зависимость $\operatorname{Re} \lambda_{i}$ (а) и $\operatorname{Im} \lambda_{i}$ (б) от $\beta$ для $f=1$ и $k=1, g=2$.

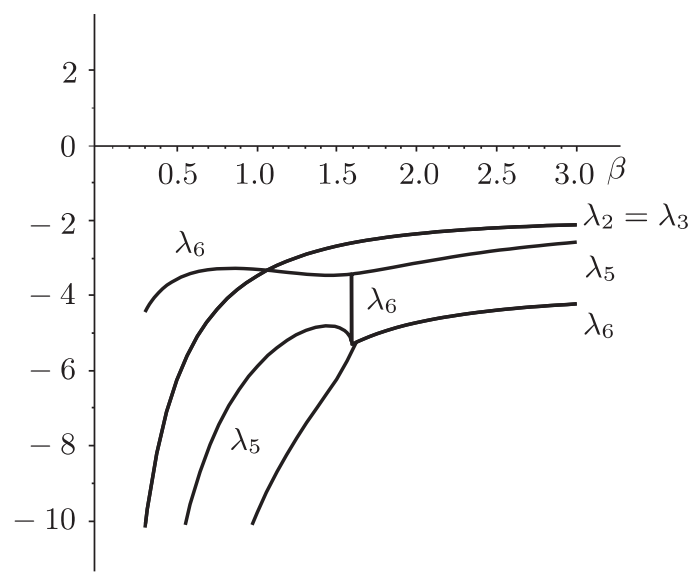

a

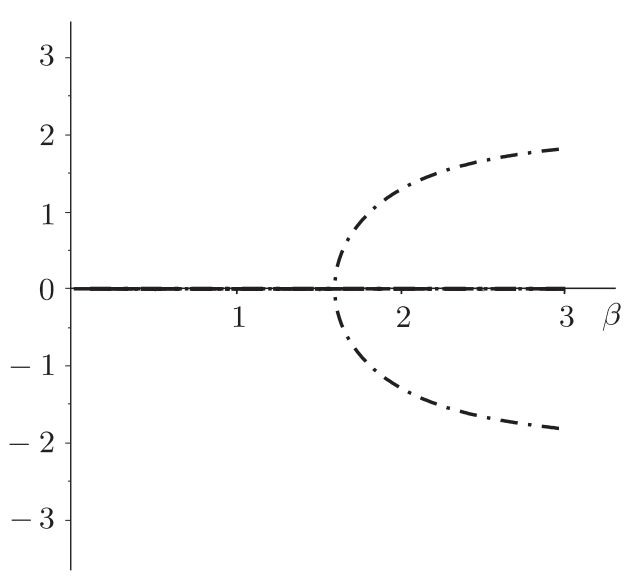

б

Рис. 7. Зависимость $\operatorname{Re} \lambda_{i}$ (а) и $\operatorname{Im} \lambda_{i}$ (б) от $\beta$ для $f=1$ и $k=2, g=2$.

в системе (см. пример на рис. 2б) стационарное состояние динамики матрицы плотности может быть неединственным (более того, характер динамики может сильно зависеть от начального состояния). Обсудим для рассматриваемой модели аналог темного состояния. Такие состояния для динамики матрицы плотности в стохастическом пределе рассматривались в работе [5]. Возьмем чистое состояние вида

$$
\varphi=\frac{1}{\sqrt{2}}(|2\rangle-|3\rangle),
$$

отвечающее матрице плотности

$$
\rho=\frac{1}{2}(|2\rangle\langle 2|+| 3\rangle\langle 3|-| 2\rangle\langle 3|-| 3\rangle\langle 2|) .
$$


Подставляя такую матрицу плотности в генератор динамики (для примера динамики с вырождением), получим нуль, поскольку

$$
\sum_{b, d=2,3} \rho_{b d}=0, \quad \sum_{b, d=2,3}|d\rangle\langle b|=(|2\rangle+|3\rangle)(\langle 2|+\langle 3|),
$$

а векторы $|2\rangle+|3\rangle,|2\rangle-|3\rangle$ ортогональны. Поэтому такое состояние является стационарным для изучаемой динамики. Возникнув, оно блокирует передачу возбуждения на сток.

\section{6. ДИНАМИКА МОДЕЛИ}

Обсудим динамику для введенного выше генератора, отвечающего квантовому графу на рис. 1. Начальное состояние системы выберем равным $|1\rangle$, т. е. матрица плотности в начальный момент времени равна $|1\rangle\langle 1| \otimes R \otimes r$, где $R$ есть гауссово состояние колебательных степеней свободы и $r$ - вакуумное состояние стока. Мы будем рассматривать динамику как комбинацию переходов между слоями квантового графа, описывающуюся генераторами $\theta_{1}, \theta_{2}, \theta_{3}$.

Стационарное решение для $\theta_{1}$. Обсудим вид стационарного решения $\theta_{1}(\rho)=0$ для первой части генератора. Подставим общий вид матрицы плотности

$$
\rho=\sum_{m n} \rho_{m n}|m\rangle\langle n|
$$

в генератор $\theta_{1}$, получим

$$
\begin{aligned}
\theta_{1}(\rho)= & \sum_{b, d=2}^{N}\left(2 \gamma_{\text {re }}^{-}\left(\rho_{11}|d\rangle\langle b|-\frac{1}{2} \delta_{b d}\left(\sum_{m} \rho_{m 1}|m\rangle\left\langle 1\left|+\sum_{n} \rho_{1 n}\right| 1\right\rangle\langle n|\right)\right)+\right. \\
& +2 \gamma_{\mathrm{re}}^{+}\left(\rho_{b d}|1\rangle\langle 1|-\frac{1}{2}\left(\sum_{m} \rho_{m d}|m\rangle\left\langle b\left|+\sum_{n} \rho_{b n}\right| d\right\rangle\langle n|\right)\right)- \\
& -i \gamma_{\mathrm{im}}^{-} \delta_{b d}\left(\sum_{m} \rho_{m 1}|m\rangle\left\langle 1\left|-\sum_{n} \rho_{1 n}\right| 1\right\rangle\langle n|\right)+ \\
& \left.+i \gamma_{\mathrm{im}}^{+}\left(\sum_{m} \rho_{m d}|m\rangle\left\langle b\left|-\sum_{n} \rho_{b n}\right| d\right\rangle\langle n|\right)\right) .
\end{aligned}
$$

Приравнивая (для стационарного решения) к нулю члены при $|1\rangle\langle b|$ для каждого $b=2, \ldots, N$, получим

$$
\left(-\gamma_{\mathrm{re}}^{-}+i \gamma_{\mathrm{im}}^{-}\right)(N-1) \rho_{1 b}+\left(-\gamma_{\mathrm{re}}^{+}+i \gamma_{\mathrm{im}}^{+}\right) \sum_{j=2}^{N} \rho_{1 j}=0 .
$$

Если просуммировать эти равенства по $b=2, \ldots, N$, то получим $\sum_{j=2}^{N} \rho_{1 j}=0$, откуда (подставляя это равенство в исходное уравнение) мы имеем $\rho_{1 b}=0$ для всех $b=2, \ldots, N$.

Приравнивая к нулю члены при $|1\rangle\langle 1|$, имеем

$$
-2 \gamma_{\mathrm{re}}^{-}(N-1) \rho_{11}+2 \gamma_{\mathrm{re}}^{+} \sum_{b, d=2}^{N} \rho_{b d}=0 .
$$


Приравнивая к нулю члены при $|d\rangle\langle b|$ для $b, d=2, \ldots, N$, получаем

$$
2 \gamma_{\mathrm{re}}^{-} \rho_{11}-\gamma_{\mathrm{re}}^{+} \sum_{j=2}^{N}\left(\rho_{d j}+\rho_{j b}\right)+i \gamma_{\mathrm{im}}^{+} \sum_{j=2}^{N}\left(\rho_{d j}-\rho_{j b}\right)=0 .
$$

Двойное суммирование по $b$ и $d$ этих уравнений дает (17), т. е. уравнение (17) есть следствие (18). Частное решение уравнения (18) получится, если положить равными друг другу $\rho_{b d}$ для всех $b, d=2, \ldots, N$ (такое частное решение неединственно [5]). Тогда для этого решения мы имеем

$$
\gamma_{\mathrm{re}}^{-} \rho_{11}=(N-1) \gamma_{\mathrm{re}}^{+} \rho_{d b} .
$$

При подстановке в $\theta_{2}$ проектор $|1\rangle\langle 1|$ обращается в нуль (поскольку отсутствуют соответствующие переходы в квантовом графе). Остальная часть полученного стационарного решения для $\theta_{1}$ равна (с точностью до нормировки)

$$
\sum_{i, j=2}^{N}|i\rangle\langle j|=\left[\sum_{i=2}^{N}|i\rangle\right] \otimes\left[\sum_{j=2}^{N}\langle j|\right]=|\psi\rangle\langle\psi|,
$$

т. е. это чистое состояние.

Подстановка состояния $|1\rangle\langle 1|$ в $\theta_{1}$. Рассмотрим действие генератора $\theta_{1}$ в одномерном семействе матриц плотности, состоящем из смесей состояний $|1\rangle\langle 1|$ и $(20)$,

$$
\rho=\rho_{11}|1\rangle\left\langle 1\left|+\sum_{i, j=2}^{N} \rho_{i j}\right| i\right\rangle\langle j|
$$

Получим

$$
\begin{aligned}
\theta_{1}(\rho)= & 2 \gamma_{\mathrm{re}}^{-} \rho_{11}\left(\sum_{b, d=2}^{N}|d\rangle\langle b|-(N-1)| 1\rangle\langle 1|\right)+ \\
& +2 \gamma_{\mathrm{re}}^{+} \rho_{b d}\left((N-1)^{2}|1\rangle\left\langle 1\left|-(N-1) \sum_{b, d=2}^{N}\right| d\right\rangle\langle b|\right) .
\end{aligned}
$$

Таким образом, генерируемая $\theta_{1}$ динамика сохраняет это подпространство. Следовательно, начальное состояние $|1\rangle\langle 1|$ под действием $\theta_{1}$ будет релаксировать к описанному выше частному стационарному состоянию (смеси состояний $|1\rangle\langle 1|$ и $|\psi\rangle\langle\psi|$, удовлетворяющей уравнению (19)).

Подстановка состояния (20) в $\theta_{2}$. Мы показали, что динамика, порождающаяся генератором $\theta_{1}$, переводит начальное состояние $|1\rangle\langle 1|$ в его смесь с чистым состоянием $|\psi\rangle\langle\psi|$, которое является комбинацией состояний промежуточного слоя квантового графа. Следующая стадия динамики состоит в распаде такого состояния на стоки.

Подставляя состояние (20) во вторую часть $\theta_{2}$ генератора, получим

$$
\begin{gathered}
\theta_{2}(\rho)=\sum_{\substack{a=2, \ldots, N, b=N+1, \ldots, N+M}} \sum_{\substack{c=2, \ldots, N, d=N+1, \ldots, N+M}} e^{-2 \pi i a(b-N-1) /(N-1)} e^{2 \pi i c(d-N-1) /(N-1)} \times \\
\times\left(2 \eta_{\mathrm{re}}^{-}\left(\langle c|\rho| a\rangle|d\rangle\langle b|-\frac{1}{2} \delta_{b d}\{\rho,|a\rangle\langle c|\}\right)-i \eta_{\mathrm{im}}^{-} \delta_{b d}[\rho,|a\rangle\langle c|]\right)=
\end{gathered}
$$




$$
\begin{aligned}
& =\sum_{\substack{a=2, \ldots, N, b=N+1, \ldots, N+M}} \sum_{\begin{array}{c}
c=2, \ldots, N, \\
d=N+1, \ldots, N+M
\end{array}} e^{-2 \pi i a(b-N-1) /(N-1)} e^{2 \pi i c(d-N-1) /(N-1)} \times \\
& \times\left(2 \eta_{\mathrm{re}}^{-}\left(|d\rangle\langle b|-\frac{1}{2} \delta_{b d}\left(\sum_{i=2}^{N}|i\rangle\left\langle c\left|+\sum_{j=2}^{N}\right| a\right\rangle\langle j|\right)\right)-i \eta_{\mathrm{im}}^{-} \delta_{b d}\left(\sum_{i=2}^{N}|i\rangle\left\langle c\left|-\sum_{j=2}^{N}\right| a\right\rangle\langle j|\right)\right) .
\end{aligned}
$$

Поскольку сумма всех корней из единицы равна нулю (при суммировании по состояниям $a, c$ второго слоя; напомним, что $M<N)$, первый член вышеприведенного генератора (содержащий $|d\rangle\langle b|$ ) обращается в нуль, кроме случая $b=d=N+1$. Аналогично, остальные члены не обращаются в нуль, только если $b=d=N+1$. Мы получим, что весь сток будет направлен на первое состояние стока, а генератор будет действовать как

$$
(N-1)^{2} 2 \eta_{\text {re }}^{-}|N+1\rangle\left\langle N+1\left|-(N-1) 2 \eta_{\text {re }}^{-} \sum_{i, j=2}^{N}\right| i\right\rangle\langle j|,
$$

т. е. все матричные элементы матрицы плотности будут убывать равномерно и идти в сток в состояние $|N+1\rangle$. Таким образом, за счет интерференции между состояниями $|2\rangle, \ldots,|N\rangle$ промежуточного слоя квантового графа сток в состоянии $|N+1\rangle$ будет усилен, а остальные стоки подавлены. Эффект подавления альтернативных стоков есть аналог рассмотренного выше эффекта темного состояния (для состояний во втором слое квантового графа).

Для доказательства того что динамика, порождающаяся генератором $\theta_{2}$, состоит в стоке в состоянии $|N+1\rangle$, рассмотрим действие генератора $\theta_{2}$ в одномерном семействе матриц плотности, состоящем из смесей состояний $|N+1\rangle\langle N+1|$ и (20):

$$
\begin{aligned}
\theta_{2}(\rho)= & \sum_{a, c=2}^{N}\left(2 \eta_{\mathrm{re}}^{-} \rho_{c a}\left(|N+1\rangle\langle N+1|-\frac{1}{2}\left(\sum_{i=2}^{N}|i\rangle\left\langle c\left|+\sum_{j=2}^{N}\right| a\right\rangle\langle j|\right)\right)+\right. \\
& \left.+2 \eta_{\mathrm{re}}^{+} \rho_{N+1, N+1}\left(|a\rangle\left\langle c\left|-\delta_{a c}\right| N+1\right\rangle\langle N+1|\right)-i \eta_{\mathrm{im}}^{-}\left(\sum_{i=2}^{N}|i\rangle\left\langle c\left|-\sum_{j=2}^{N}\right| a\right\rangle\langle j|\right)\right)= \\
= & \left(2 \eta_{\mathrm{re}}^{-} \rho_{c a}\left((N-1)^{2}|N+1\rangle\left\langle N+1\left|-(N-1) \sum_{a, c=2}^{N}\right| a\right\rangle\langle c|\right)+\right. \\
& +2 \eta_{\mathrm{re}}^{+} \rho_{N+1, N+1}\left(\sum_{a, c=2}^{N}|a\rangle\langle c|-(N-1)| N+1\rangle\langle N+1|\right) .
\end{aligned}
$$

Таким образом, генератор $\theta_{2}$ сохраняет подпространство, натянутое на состояния $|N+1\rangle\langle N+1|$ и $(20)$, поэтому вышеизложенные выводы о динамике матрицы плотности являются строгими.

Особенностью полученного результата является квадратичная зависимость, т. е. пропорциональность $(N-1)^{2}$, стока $(21)$ от числа частиц в промежуточном слое квантового графа. Тот факт, что подобная квадратичная зависимость для генератора динамики матрицы плотности многочастичной системы возможна, был отмечен в работе [4]. При выводе мы не принимали во внимание нормировку, согласно которой используемое состояние нужно разделить на $N-1$. Таким образом, с учетом 
нормировки мы получаем квантовый эффект ускорения за счет конструктивной интерференции для транспорта экситонов: скорость пропорциональна первой степени числа частиц $N-1$ в промежуточном слое квантового графа.

ЗАмечАниЕ 1. Отмеченный эффект можно интерпретировать как эффект прохождения квантовой частицей в многощелевом эксперименте через все промежуточные щели одновременно. Если бы частица проходила только через одну щель, то скорость прохождения не зависела от количества щелей, а для представленной модели показано, что скорость прохождения пропорциональна количеству щелей $N-1$.

Мы обсуждаем интерференцию на языке линдбладовских генераторов для матрицы плотности. При этом интерференция имеет место между вкладами в диссипативную часть генератора, которые отвечают прохождениям экситона в стоки через различные состояния второго слоя квантового графа (состояния $|2\rangle, \ldots,|N\rangle)$. Для наличия интерференции обязательным является вырождение генератора во втором слое квантового графа, т. е. для переходов между вторым и третьим слоями квантового графа должно присутствовать много вкладов в генератор, способных интерферировать между собой. Вырождение гамильтониана модели в третьем слое (содержащем стоки) является несущественным и введено для простоты.

\section{7. ЗАКЛЮЧЕНИЕ}

В настоящей работе рассмотрена модель экситонного транспорта, основанная на квантовой интерференции, которая описывается мастер-уравнением, полученным методом стохастического предела. При этом путем подбора параметров модели удается усилить перенос в избранный сток, а также подавить нежелательные альтернативные стоки. Для интерференционной картины важным является вырождение гамильтониана во втором слое квантового графа (в состояниях $|2\rangle, \ldots,|N\rangle$ ). Такое вырождение позволяет генерировать состояние, представляющее собой когерентную комбинацию состояний второго слоя. Вырождение в третьем слое (содержащем стоки) является несущественным. Более того, можно рассмотреть модель, для которой когерентное состояние во втором слое квантового графа может устанавливаться при помощи динамики, не описывающейся методом стохастического предела (например, не удовлетворяющей условию слабой связи), а распад такого состояния на стоки с соответствующей интерференционной картиной может описываться стохастическим пределом. Для наличия интерференции важна именно когерентность состояния во втором слое.

Есть указания на то, что такие состояния действительно существуют в фотосинтетических комплексах [8]-[10]. Предложенная в настоящей статье модель показывает, что эту когерентность можно использовать для повышения эффективности экситонного транспорта (а значит, эффективности фотосинтеза).

Суммируем изложенное: мы показали, что для описанной выше динамики на квантовом графе начальное состояние $|1\rangle\langle 1|$ будет распадаться в сток $|N+1\rangle\langle N+1|$ со скоростью, пропорциональной числу $N-1$ узлов в промежуточном слое квантового графа. При этом распад через альтернативные стоки $|N+2\rangle\langle N+2|, \ldots| N+M\rangle,\langle N+M|$ будет подавлен за счет квантовой интерференции.

Благодарности. Исследование выполнено за счет гранта Российского научного фонда (проект № 14-11-00687). 


\section{Список литературы}

[1] L. Accardi, L. Y. Gang, I. Volovich, Quantum Theory and Its Stochastic Limit, Springer, Berlin, 2002.

[2] V. May, O. Kühn, Charge and Energy Transfer Dynamics in Molecular Systems, Wiley-VCH, Weinheim, 2011.

[3] L. Accardi, S. V. Kozyrev, I. V. Volovich, Phys. Rev. A, 56:4 (1997), 2557-2562, arXiv: quant-ph/9706021.

[4] L. Accardi, S. V. Kozyrev, "Lectures on quantum interacting particle systems", Quantum Interacting Particle Systems (Trento, Italy, September 23-29, 2000), Quantum Probability and White Noise Analysis, 14, eds. L. Accardi, F. Fagnola, World Sci., Singapore, 2002, $1-195$.

[5] L. Accardi, S. V. Kozyrev, Internat. J. Theoret. Phys., 45:4 (2006), 661-678, arXiv: quant-ph/0303185.

[6] G.D. Scholes, G.R. Fleming, A. Olaya-Castro, R. van Grondelle, Nature Chem., 3:10 (2011), 763-774.

[7] M. Ohya, I. Volovich, Mathematical Foundations of Quantum Information and Computation and Its Applications to Nano- and Bio-systems, Springer, Dordrecht, 2011.

[8] G. S. Engel, T. R. Calhoun, E. L. Read, T.-K. Ahn, T. Mancal, Y.-C. Cheng, R. E. Blankenship, G. R. Fleming, Nature, 446:7137 (2007), 782-786.

[9] A. Ishizaki, G. R. Fleming, Proc. Natl. Acad. Sci. USA, 106:41 (2009), 17255-17260.

[10] E. Collini, C. Y. Wong, K. E. Wilk, P. M. G. Curmi, P. Brumer, G. D. Scholes1, Nature, 463:7281 (2010), 644-647.

[11] A. W. Chin, A. Datta, F. Caruso, S. F. Huelga, M. B. Plenio, New J. Phys., 12 (2010), 065002, 16 pp., arXiv: 0910.4153.

Поступила в редакцию 25.11.2014 